Article

\title{
Complex Modified Projective Synchronization of Fractional-Order Complex-Variable Chaotic System with Unknown Complex Parameters
}

\author{
Ruoxun Zhang ${ }^{1, *}$, Shiwen Feng ${ }^{1}$ and Shiping Yang ${ }^{2}$ \\ 1 College of Primary Education, Xingtai University, Xingtai 054001, China; xtxyfsw@126.com \\ 2 College of Physics Science and Information Engineering, Hebei Normal University, Shijiazhuang 050016, \\ China; yangship@hebtu.edu.cn \\ * Correspondence: xtzhrx@126.com; Tel.: +86-13292167929
}

Received: 17 March 2019; Accepted: 15 April 2019; Published: 17 April 2019

\begin{abstract}
This paper investigates the problem of complex modified projective synchronization (CMPS) of fractional-order complex-variable chaotic systems (FOCCS) with unknown complex parameters. By a complex-variable inequality and a stability theory for fractional-order nonlinear systems, a new scheme is presented for constructing CMPS of FOCCS with unknown complex parameters. The proposed scheme not only provides a new method to analyze fractional-order complex-valued systems but also significantly reduces the complexity of computation and analysis. Theoretical proof and simulation results substantiate the effectiveness of the presented synchronization scheme.
\end{abstract}

Keywords: complex modified projective synchronization (CMPS); complex modified projective synchronization (CMPS); complex-variable chaotic system; unknown complex parameters

\section{Introduction}

In the past twenty years, the application of fractional calculus has become a focus of attention, since fractional derivatives can more accurately describe the actual physical model. So, it has become an efficient and an excellent tool in physics, mathematical science, chemistry, control engineering, finance, signal processing and other fields [1-6]. Meanwhile, chaotic dynamics and synchronization of fractional-order nonlinear systems have aroused tremendous attention of many researchers. Many excellent results have been obtained and some types of synchronization have been presented [7-22]. In various synchronizations, modified projective synchronization (MPS) refers to the master system and slave system being synchronized to a constant scaling diagonal matrix. In secure communication, this scaling feature extends the binary digital to M-nary digital for faster transmission. It is quite clear that complete synchronization (CS), ant-synchronization (AS) and projective synchronization (PS) are each special cases of MPS. Therefore, the study of MPS has important theoretical significance and application prospect.

The above-mentioned works mainly investigated the fractional-order systems with real variables, not involving complex variables. It is well known that complex variables, which double the number of variables, can generate complicated dynamical behaviors, enhance anti-attack ability and achieve higher transmission efficiency [23-25]. The complex-variable systems can be widely applied to describe a variety of physical phenomena, for example, atomic polarization amplitudes, electric fields, population inversion $[26,27]$, detuned laser systems, amplitudes of electromagnetic fields, thermal convections of liquid flows [28-30], etc. Many researchers have taken included complex into variables in fractional-order systems and have investigated dynamic behavior, stability, stabilization and synchronization of fractional-order complex-variable chaotic systems (FOCCS) in recent years [31-37]. 
Especially, as a new synchronization phenomenon, the complex modified projective synchronization (CMPS) was first introduced by Mahmoud et al. [38], which can be seen as an extension of MPS. That is, in complex space, two complex-variable systems can achieve MPS with desired complex scaling factors. As the complex scaling factor is arbitrary and more unpredictable than the real scaling factor, the capacity of the transmitted message is doubled and the safety is greatly strengthened [39]. So, study on CMPS of fractional complex-variable chaotic systems is of great theoretical and practical significance. However, in the existing literature, parameters of the FOCCS are real and known in priori. In fact, in many practical engineering situations, most of system parameters cannot be accurately determined in advance and chaos synchronization will be destroyed with these uncertain factors. Meanwhile, the complex parameter is more complex and unpredictable than the real parameter, and if the initial values are real numbers, the existing FOCCS $[24,31,32]$ will become a fractional-order real-variable chaotic system. Hence, it is an important problem to realize CMPS of FOCCS with unknown complex parameters.

Inspired by the above discussions, the CMPS problem of FOCCS with unknown complex parameters is investigated in this paper. First, we present a stability theory for fractional-order uncertain nonlinear systems. Then, using this theory, the inequality proposed by $\mathrm{Xu}$ et al. [39] and complex analysis techniques, we realize CMPS of such systems via constructing a suitable response system. It should be noted that we deal with the synchronization problem of fractional-order uncertain complex-variable systems in the complex domain. That is to say, it is not necessary to separate the complex-variable system into its real and imaginary parts. This greatly reduces the complexity of computation and the difficulty of theoretical analysis.

Notation: $\mathbb{C}^{n}$ denotes complex $n$-dimensional space. $z \in \mathbb{C}^{n}, z^{r}, z^{i}, \bar{z}, z^{T}, z^{H}$ and $\|z\|$ represent the real part, imaginary part, conjugate, transpose, conjugate transpose and $l_{2}$-norm of $z$, respectively. For a matrix $A \in \mathbb{C}^{n \times n}, A^{H}$ denotes its conjugate transpose. $\mathbb{H}^{n \times n}$ denotes the set of $n \times n$ Hermite matrices.

\section{Preliminaries}

\subsection{Fractional Calculus}

Definition 1 [40]. The fractional integral of order $\alpha$ for a function $f$ is defined as

$$
I^{\alpha} f(t)={ }_{t_{0}} D_{t}^{-\alpha} f(t)=\frac{1}{\Gamma(\alpha)} \int_{t_{0}}^{t}(t-\tau)^{\alpha-1} f(\tau) d \tau
$$

where $t \geq t_{0}$ and $\alpha>0$.

Definition 2 [40]. Caputo's fractional derivative of order $\alpha$ for a function $f \in \mathbb{R}^{n}$ is defined by

$$
{ }_{t_{0}}^{C} D_{t}^{\alpha} f(t)=\frac{1}{\Gamma(n-\alpha)} \int_{t_{0}}^{t} \frac{f^{(n)}(\tau)}{(t-\tau)^{\alpha-n+1}} d \tau
$$

where $t \geq t_{0}$ and $n$ is a positive integer such that $n-1<\alpha<1$.

Lemma 1. [41] Let $x(t) \in \mathbb{R}^{n}$ be a continuous and derivable vector function. Then, for any time instant $t \geq t_{0}$ and $\alpha \in(0,1)$,

$$
\frac{1}{2^{t_{0}}} D_{t}^{\alpha}\left[x^{T}(t) x(t)\right] \leq x^{T}(t)_{t_{0}}^{C} D_{t}^{\alpha} x(t) .
$$

Corollary 1. For a scalar derivable function $\varphi(t)$ and a constants $C$, we have

$$
\frac{1}{2}{ }^{t_{0}} D_{t}^{\alpha}(\varphi(t)-C)^{2} \leq(\varphi(t)-C)_{t_{0}}^{C} D_{t}^{\alpha} \varphi(t) .
$$


Lemma 2. [42] Let $z \in \mathbb{C}^{n}$ be a differentiable complex-valued vector. Then, $\forall t \geq t_{0}$ and $\alpha \in(0,1$ ], the following inequality holds

$$
{ }_{t_{0}}^{C} D_{t}^{\alpha} z^{H}(t) P z(t) \leq z^{H}(t) P_{t_{0}}^{C} D_{t}^{\alpha} z(t)+\left({ }_{t_{0}}^{C} D_{t}^{\alpha} z(t)\right)^{H} P z(t) .
$$

where $P \in \mathbb{C}^{n \times n}$ is a constant positive definite Hermitian matrix.

Lemma 3. (Stability theory for fractional-order system) Let $V_{1}(t)$ be a uniformly continuous and derivable Lyapunov function, $V_{2}(t)$ be a derivable and nonnegative function. If

$$
V(t)=V_{1}(t)+V_{2}(t)
$$

and

$$
{ }_{0}^{C} D_{t}^{\alpha} V(t) \leq-\theta V_{1}(t)
$$

where $\theta$ is a positive constant. Then $\lim _{t \rightarrow \infty} V_{1}(t)=0$.

Proof. By $\alpha$-integrating (7), we have:

$$
V(t)-V(0) \leq-\theta I^{\alpha} V_{1}(t) \leq 0
$$

Thus, $V(t) \leq V(0), t \geq 0$. From (6), we can obtain that $V_{1}(t)$ and $V_{2}(t)$ are bounded.

Next, we adopt contradiction to prove $\lim _{t \rightarrow \infty} V_{1}(t)=0$ using the idea of Theorem 1 in [43].

Suppose that $V_{1}(t) \neq 0$ as $t \rightarrow \infty$. Then there exists a monotone increasing sequence $\left(t_{k}\right)_{k \in N^{+}}$ $\left(t_{k} \rightarrow \infty\right.$ as $\left.k \rightarrow \infty\right)$ and a positive constant $\varepsilon>0$ such that $V_{1}\left(t_{k}\right)>\varepsilon$. Since the uniform continuity of $V_{1}(t)$, for the given $\varepsilon, \exists \delta>0\left(\delta \leq \inf _{j \in N^{+}}\left\{t_{j+1}-t_{j}\right\}\right.$, which implies that the intervals $\left[t_{k}, t_{k}+\delta\right]$ are nonoverlapping) such that $\left|V_{1}\left(t_{k}\right)-V_{1}(t)\right| \leq \varepsilon / 2$. Then, for any $t \in\left[t_{k}, t_{k}+\delta\right]$ it follows that $V_{1}(t)=V_{1}\left(t_{k}\right)-V_{1}\left(t_{k}\right)+V_{1}(t) \geq\left|V_{1}\left(t_{k}\right)\right|-\left|V_{1}\left(t_{k}\right)-V_{1}(t)\right|>\varepsilon / 2$. Thus, for any $k=1,2,3, \ldots$, from (7), we have:

$$
\begin{aligned}
V\left(t_{k}+\delta\right)-V(0) & \leq \frac{-\theta}{\Gamma(\alpha)} \int_{0}^{t_{k}+\delta} \frac{V_{1}(\tau)}{\left(t_{k}+\delta-\tau\right)^{1-\alpha}} d \tau \\
& =\frac{-\theta}{\Gamma(\alpha)}\left[\int_{0}^{t_{1}}+\int_{t_{1}}^{t_{1}+\delta}+\int_{t_{1}+\delta}^{t_{2}}+\int_{t_{2}}^{t_{2}+\delta}+\int_{t_{2}+\delta}^{t_{3}}+\int_{t_{3}}^{t_{3}+\delta}+\cdots+\int_{t_{k}}^{t_{k}+\delta}\right] \frac{V_{1}(\tau)}{\left(t_{k}+\delta-\tau\right)^{1-\alpha}} d \tau \\
& \leq \frac{-\theta}{\Gamma(\alpha)}\left[\int_{t_{1}}^{t_{1}+\delta}+\int_{t_{2}}^{t_{2}+\delta}+\int_{t_{3}}^{t_{3}+\delta}+\cdots+\int_{t_{k}}^{t_{k}+\delta}\right] \frac{V_{1}(\tau)}{\left(t_{k}+\delta-\tau\right)^{1-\alpha}} d \tau \\
& \leq \frac{-\theta \varepsilon}{2 \Gamma(\alpha)}\left[\int_{t_{1}}^{t_{1}+\delta}+\int_{t_{2}}^{t_{2}+\delta}+\int_{t_{3}}^{t_{3}+\delta}+\cdots+\int_{t_{k}}^{t_{k}+\delta}\right]\left(t_{k}+\delta-\tau\right)^{\alpha-1} d \tau \\
& =\frac{-\theta \varepsilon}{2 \Gamma(\alpha)} \sum_{j=1}^{k} \int_{t_{j}}^{t_{j}+\delta}\left(t_{k}+\delta-\tau\right)^{\alpha-1} d \tau
\end{aligned}
$$

Given that $\left(t_{k}+\delta-\tau\right)^{\alpha-1} \geq\left(t_{k}+\delta-t_{j}\right)^{\alpha-1}$ for all $\tau \in\left[t_{j}, t_{j}+\delta\right]$ results in:

$$
\begin{aligned}
V\left(t_{k}+\delta\right)-V(0) & \leq \frac{-\theta \varepsilon}{2 \Gamma(\alpha)} \sum_{j=1}^{k} \int_{t_{j}}^{t_{j}+\delta}\left(t_{k}+\delta-\tau\right)^{\alpha-1} d \tau \leq \frac{-\theta \varepsilon}{2 \Gamma(\alpha)} \sum_{j=1}^{k} \int_{t_{j}}^{t_{j}+\delta}\left(t_{k}+\delta-t_{j}\right)^{\alpha-1} d \tau \\
& =\frac{-\theta \varepsilon \delta}{2 \Gamma(\alpha)} \sum_{j=1}^{k}\left(t_{k}+\delta-t_{j}\right)^{\alpha-1} \leq \frac{-\theta \varepsilon \delta}{2 \Gamma(\alpha)} \sum_{j=1}^{k}\left(t_{k}+\delta-t_{1}\right)^{\alpha-1} \\
& =\frac{-\theta \varepsilon \delta}{2 \Gamma(\alpha)} \frac{k}{\left(t_{k}+\delta-t_{1}\right)^{1-\alpha}} \leq \frac{-\theta \varepsilon \delta}{2 \Gamma(\alpha)} \frac{k}{(k d)^{1-\alpha}}=\frac{-\theta \varepsilon \delta}{2 \Gamma(\alpha)} \frac{k^{\alpha}}{(d)^{1-\alpha}}
\end{aligned}
$$

where $d=\sup _{j \in N, 2 \leq j \leq k}\left\{t_{j}-t_{j-1}\right\}$ (since $V_{1}(t)$ is a uniformly continuous function and assumed $V_{1}(t) \neq 0$, as $t \rightarrow \infty, d$ is bounded). Obviously, $V\left(t_{k}+\delta\right)-V(0) \leq \frac{-\theta \varepsilon \delta}{2 \Gamma(\alpha)} \frac{k^{\alpha}}{(d)^{1-\alpha}} \rightarrow-\infty$ as $k \rightarrow \infty$, which contradicts with $V(t) \geq 0$. Therefore, $\lim _{t \rightarrow \infty} V_{1}(t)=0$. 
Remark 1. Lemma 3 provides a stability criterion for the fractional-order nonlinear uncertain system by choosing a Lyapunov function that includes two parts $V_{1}(t)$ and $V_{2}(t)$.

Remark 2. Lemma 3 provides a Lyapunov-based adaptive control method for stability analysis and synchronization of fractional-order systems (FOS).

Remark 3. Lemma 3 is suitable for verifying the stability and stabilization controller design of FOS with unknown parameters and external disturbances.

Remark 4. If the FOS has no uncertainties, then Lemma 3 is still valid.

Lemma 4. [44] For the fractional-order complex-variable systems

$$
{ }_{0}^{C} D_{t}^{\alpha} z(t)=h(z(t))
$$

where $0<\alpha<1, z(t)=\left(z_{1}, z_{2}, \cdots, z_{n}\right)^{T} \in \mathbb{C}^{n}$ is the system complex state vector, $h \in \mathbb{C}^{n}$ is a continuous nonlinear function complex vector, which satisfies the globally Lipschitz continuity condition in the complex domain. Let $z(t)=0$ be an equilibrium point of system (1), and let $V_{1}(t)=z^{H}(t) z(t)$ and $V_{2}(z(t)) \geq 0$ are continuously differentiable functions. If

$$
V(t)=V_{1}(t)+V_{2}(z(t))
$$

and

$$
{ }_{0}^{t} D_{t}^{\alpha} V(t) \leq-\theta V_{1}(t)
$$

where $\theta$ is a positive constant, then $z(t)=0$ is asymptotically stable.

Lemma 5. [45,46] For all $z \in \mathbb{C}^{n}, B \in \mathbb{H}^{n \times n}$, there exists a positive constant $l_{1}$ such that the following inequality holds:

$$
\lambda_{\min }(B) z^{H} z \leq z^{H} B z \leq \lambda_{\max }(B) z^{H} z
$$

where $\lambda_{\min }$ and $\lambda_{\max }$ are the minimum and maximum eigenvalue of $B$.

Lemma 6. [44,46] For the Lipschitz continuous function $f \in \mathbb{C}^{n}$, there exists a positive constant $l_{2}$ such that the following inequality holds:

$$
(z-w)^{H}[f(z)-f(w)]+[f(z)-f(w)]^{H}(z-w) \leq l_{2}(z-w)^{H}(z-w)
$$

\subsection{System Model}

We consider a kind of fractional-order complex-variable chaotic drive and response systems

$$
\begin{aligned}
& { }_{0}^{C} D_{t}^{\alpha} z(t)=F z(t)+f(z(t)) \\
& { }_{0}^{C} D_{t}^{\alpha} w(t)=H w(t)+h(w(t))
\end{aligned}
$$

where $0<\alpha<1, z(t)=\left(z_{1}, z_{2}, \cdots, z_{n}\right)^{T} \in \mathbb{C}^{n}$ and $w(t)=\left(w_{1}, w_{2}, \cdots, w_{n}\right)^{T} \in \mathbb{C}^{n}$ are the system complex state vectors, $f \in \mathbb{C}^{n}$ and $h \in \mathbb{C}^{n}$ are continuous complex nonlinear function vectors, $F \in \mathbb{C}^{n \times n}$ and $H \in \mathbb{C}^{n \times n}$ are complex or real parameter matrices. 
Definition 3. [38] For given drive system (8) and response system (9), it is said to achieve CMPS if

$$
\lim _{t \rightarrow \infty}\|w(t)-M z(t)\|=0
$$

where $M \in \mathbb{C}^{n \times n}$ is the desired scaling diagonal complex matrix.

Remark 5. Obviously, some known synchronization ways are the special cases of CMPS, such as CS, AS, PS and MPS.

\section{Main Results}

We consider a kind of FOCCS with unknown complex parameters as

$$
{ }_{0}^{C} D_{t}^{\alpha} z(t)=A z(t)+f(z(t))=g(z(t)) \theta+f(z(t))
$$

where $0<\alpha<1, z(t)=\left(z_{1}, z_{2}, \cdots, z_{n}\right)^{T} \in \mathbb{C}^{n}$ is the system complex state vector, $f \in \mathbb{C}^{n}$ is a continuous nonlinear function vector, which satisfies the globally Lipschitz continuity condition in the complex domain, $A \in \mathbb{C}^{n \times n}$ is an unknown (complex or real) parameter matrix and $g \in \mathbb{C}^{n \times m}$ is a complex function matrix, and $\theta=\left(\theta_{1}, \theta_{2}, \cdots, \theta_{m}\right)^{T}$ is the system unknown complex parameter vector.

System (13) was chosen as the master system. In this case, we constructed the slave system as follows:

$$
{ }_{0}^{C} D_{t}^{\alpha} w(t)=M g\left(M^{-1} w(t)\right) \hat{\theta}+M f\left(M^{-1} w(t)\right)+u(t)
$$

where $w(t)=\left(w_{1}, w_{2}, \cdots, w_{n}\right)^{T}$ is the complex state vector, $\hat{\theta} \in \mathbb{C}^{m}$ represents the estimate vector of the unknown vector $\theta, M \in \mathbb{C}^{n \times n}$ is the desired scaling diagonal complex matrix, and $u(t)=$ $\left(u_{1}(t), u_{2}(t), \cdots, u_{n}(t)\right)^{T}$ is the controller, to be determined.

Remark 2. From (13), it follows that $g(z(t)) \theta=A z(t)$ and $g\left(M^{-1} w(t)\right) \theta=A M^{-1} w(t)$.

Theorem 1. Asymptotically CMPS of systems (13) and (14) can be achieved under adaptive controller

$$
u(t)=-k e(t)
$$

and the complex update laws:

$$
\begin{gathered}
{ }_{0}^{C} D_{t}^{\alpha} k=\sigma e^{H}(t) e(t) \\
{ }_{0}^{C} D_{t}^{\alpha} e_{\theta}={ }_{0}^{C} D_{t}^{\alpha} \hat{\theta}=-\eta g^{H}\left(M^{-1} w(t)\right) M^{H} e(t)
\end{gathered}
$$

where $e(t)=w(t)-M z(t)$ is the error vector, $e_{\theta}=\hat{\theta}-\theta$ is the parameter error, $\theta, \eta$ are two arbitrary positive constants.

Proof. From the error vector and systems (13) and (14), it follows:

$$
\begin{aligned}
{ }_{0}^{C} D_{t}^{\alpha} e(t) & ={ }_{0}^{C} D_{t}^{\alpha} w(t)-M_{0}^{C} D_{t}^{\alpha} z(t) \\
& =M g\left(M^{-1} w(t)\right) \hat{\theta}+M f\left(M^{-1} w(t)\right)-M[g(z(t)) \theta+f(z(t))]-k e(t) \\
& =M g\left(M^{-1} w(t)\right)(\hat{\theta}-\theta)+M\left[g\left(M^{-1} w(t)\right)-g(z(t))\right] \theta+M\left[f\left(M^{-1} w(t)\right)-f(z(t))\right]-k e(t) \\
& =M g\left(M^{-1} w(t)\right) e_{\theta}+M\left[g\left(M^{-1} w(t)\right)-g(z(t))\right] \theta+M\left[f\left(M^{-1} w(t)\right)-f(z(t))\right]-k e(t)
\end{aligned}
$$

Let us present the Lyapunov function:

$$
V(t)=V_{1}(t)+V_{2}(t)
$$

where $V_{1}(t)=e^{H}(t) e(t), V_{2}(t)=\frac{1}{\sigma}\left(k-k^{*}\right)^{2}+\frac{1}{\eta} e_{\theta}^{H}(t) e_{\theta}(t), k^{*}$ is to be determined. 
Using Lemma 1, Corollary 1 and Lemma 2, we have:

$$
\begin{aligned}
& { }_{0}^{C} D_{t}^{\alpha} V(t)={ }_{0}^{C} D_{t}^{\alpha}\left[e^{H}(t) e(t)+\frac{1}{\sigma}\left(k-k^{*}\right)^{2}+\frac{1}{\eta} e_{\theta}^{H}(t) e_{\theta}(t)\right] \\
& \leq e^{H}(t){ }_{0}^{C} D_{t}^{\alpha} e(t)+\left[{ }_{0}^{C} D_{t}^{\alpha} e(t)\right]^{H} e(t)+\frac{2}{\sigma}\left(k-k^{*}\right){ }_{0}^{C} D_{t}^{\alpha} k+\frac{1}{\eta} e_{\theta}^{H}(t){ }_{0}^{C} D_{t}^{\alpha} e_{\theta}(t)+\frac{1}{\eta}\left[{ }_{0}^{C} D_{t}^{\alpha} e_{\theta}(t)\right]^{H} e_{\theta}(t) \\
& \leq e^{H}(t)\left\{M g\left(M^{-1} w(t)\right) e_{\theta}+M\left[g\left(M^{-1} w(t)\right)-g(z(t))\right] \theta+M\left[f\left(M^{-1} w(t)\right)-f(z(t))\right]-k e(t)\right\} \\
& +\left\{M g\left(M^{-1} w(t)\right) e_{\theta}+M\left[g\left(M^{-1} w(t)\right)-g(z(t))\right] \theta+M\left[f\left(M^{-1} w(t)\right)-f(z(t))\right]-k e(t)\right\}^{H} e(t) \\
& +\frac{2}{\sigma}\left(k-k^{*}\right){ }_{0}^{C} D_{t}^{\alpha} k+\frac{1}{\eta} e_{\theta}^{H}(t){ }_{0}^{C} D_{t}^{\alpha} e_{\theta}(t)+\frac{1}{\eta}\left[{ }_{0}^{C} D_{t}^{\alpha} e_{\theta}(t)\right]^{H} e_{\theta}(t)
\end{aligned}
$$

Substituting Equations (16) and(17) into the inequality above, we further have:

$$
\begin{aligned}
& { }_{0}^{C} D_{t}^{\alpha} V(t) \\
& \leq e^{H}(t)\left\{M g\left(M^{-1} w(t)\right) e_{\theta}+M\left[g\left(M^{-1} w(t)\right)-g(z(t))\right] \theta+M\left[f\left(M^{-1} w(t)\right)-f(z(t))\right]-k e(t)\right\} \\
& +\left\{M g\left(M^{-1} w(t)\right) e_{\theta}+M\left[g\left(M^{-1} w(t)\right)-g(z(t))\right] \theta+M\left[f\left(M^{-1} w(t)\right)-f(z(t))\right]-k e(t)\right\}^{H} e(t) \\
& +2\left(k-k^{*}\right) e^{H}(t) e(t)-e_{\theta}^{H}(t) g^{H}(w(t)) e(t)-\left[g^{H}(w(t)) e(t)\right]^{H} e_{\theta}(t) \\
& =e^{H}(t) M A\left[M^{-1} w(t)-z(t)\right]+\left[M^{-1} w(t)-z(t)\right]^{H} A^{H} M^{H} e(t) \\
& +e^{H}(t) M\left[f\left(M^{-1} w(t)\right)-f(z(t))\right]+\left[f\left(M^{-1} w(t)\right)-f(z(t))\right]^{H} M^{H} e(t)-2 k^{*} e^{H}(t) e(t)
\end{aligned}
$$

From Lemma 4 and Lemma 5, we can obtain:

$$
\begin{aligned}
& e^{H}(t) M A\left[M^{-1} w(t)-z(t)\right]+\left[M^{-1} w(t)-z(t)\right]^{H} A^{H} M^{H} e(t) \\
& =e^{H}(t) M A M^{-1} e(t)+e^{H}(t)\left(M^{-1}\right)^{H} A^{H} M^{H} e(t) \\
& =e^{H}(t)\left[M A M^{-1}+\left(M^{-1}\right)^{H} A^{H} M^{H}\right] e(t) \leq L_{1} e^{H}(t) e(t)
\end{aligned}
$$

and

$$
\begin{aligned}
& e^{H}(t) M\left[f\left(M^{-1} w(t)\right)-f(z(t))\right]+\left[f\left(M^{-1} w(t)\right)-f(z(t))\right]^{H} M^{H} e(t) \\
& =\left[M^{-1} w(t)-z(t)\right]^{H} M^{H} M\left[f\left(M^{-1} w(t)\right)-f(z(t))\right] \\
& +\left[f\left(M^{-1} w(t)\right)-f(z(t))\right]^{H} M^{H} M\left[M^{-1} w(t)-z(t)\right] \\
& \leq l_{2}\left[M^{-1} w(t)-z(t)\right]^{H}\left[M^{-1} w(t)-z(t)\right] \\
& \leq l_{2} e^{H}(t)\left(M^{-1}\right)^{H} M^{-1} e(t) \leq L_{2} e^{H}(t) e(t)
\end{aligned}
$$

where $L_{1}, l_{2}$ and $L_{2}$ are three positive constants. Then, one has:

$$
{ }_{0}^{C} D_{t}^{\alpha} V(t) \leq\left(L_{1}+L_{2}-2 k^{*}\right) e^{H}(t) e(t)
$$

Let $2 k^{*}=L_{1}+L_{2}+1$, then:

$$
{ }_{0}^{C} D_{t}^{\alpha} V(t) \leq-e^{H}(t) e(t)=-V_{1}(t) .
$$

By $\alpha$-integrating (20), we have $V(t) \leq V(0)$, which implies $e^{H}(t) e(t),\left\|e_{\theta}\right\|,\|k(t)\|$ are bounded and $\left\|{ }_{0}^{C} D_{t}^{\alpha} e(t)\right\|$ is also bounded according to (18) and system (13) being chaotic. Then, from [44], $V_{1}(t)=e^{H}(t) e(t)$ is uniformly continuous. Therefore, it follows from (19) and (20) and Lemma 3 that

$$
\lim _{t \rightarrow \infty} V_{1}(t)=\lim _{t \rightarrow \infty} e^{H}(t) e(t)=0 .
$$

Therefore, the systems (13) and (14) can reach asymptotically CMPS under the adaptive control strategy (15-17). 
Remark 6. Unlike previous works, in our proposed method, the entire analysis process is performed in the complex-valued domain, and the complex function theory is used to derive synchronization conditions without separating the original complex-valued chaotic system into two real-valued systems, which reduces the complexity of analysis and computation.

Remark 7. If the system parameters are known, the update law will be reduced to (16) only.

\section{Numerical Simulations}

In this section, in order to show the effectiveness of the proposed scheme in preceding section, numerical example on fractional-order complex chaotic system will be provided. When numerically solving such systems, we adopted the Grünwald-Letnikov (G-L) method [47] using MATLAB. Lyapunov exponents of the systems were calculated by adopting the Wolf et al. algorithm [48] with some changes.

Consider the fractional-order Chen complex chaotic system with commensurate order:

$$
\left(\begin{array}{l}
{ }_{0}^{c} D_{t}^{\alpha} z_{1} \\
{ }_{0}^{C} D_{t}^{\alpha} z_{2} \\
{ }_{0}^{C} D_{t}^{\alpha} z_{3}
\end{array}\right)=\left(\begin{array}{c}
a\left(z_{2}-z_{1}\right) \\
(c-a) z_{1}+c z_{2}-z_{1} z_{3} \\
\left(\bar{z}_{1} z_{2}+z_{1} \bar{z}_{2}\right) / 2-b z_{3}
\end{array}\right)=\left(\begin{array}{ccc}
z_{2}+z_{1} & 0 & 0 \\
-z_{1} & z_{1}+z_{2} & 0 \\
0 & 0 & -z_{3}
\end{array}\right)\left(\begin{array}{l}
a \\
c \\
b
\end{array}\right)+\left(\begin{array}{c}
0 \\
-z_{1} z_{3} \\
\left(\bar{z}_{1} z_{2}+z_{1} \bar{z}_{2}\right) / 2
\end{array}\right)
$$

where $z_{1}, z_{2}, z_{3}$ are the complex state variables and $a, b, c$ are system parameters, let $a=30+i$, $b=3, c=26+0.6 i$. The maximum Lyapunov exponent (MLE) spectrum is depicted in Figure 1a, and the bifurcation diagram is presented in Figure 1b. Figure 1a,b show that system (19) is chaotic with fractional order $\alpha \in[0.832,1]$ and parameters $a=30+i, b=3, c=26+0.6 i$. When the fractional-order $\alpha=0.9$, the attractor trajectories are illustrated in Figure 2. Recently, [49] described how to perform a successful simulation and optimization, and how to synthesize the mathematical models using CMOS technology. The application of metaheuristics to optimize MLE by varying the parameters of the oscillators was discussed. Here, the MLE spectrum with varying parameter $a^{i}$ (the imaginary of $a$ ) is depicted in Figure 3a, the bifurcation diagram is presented in Figure 3b.

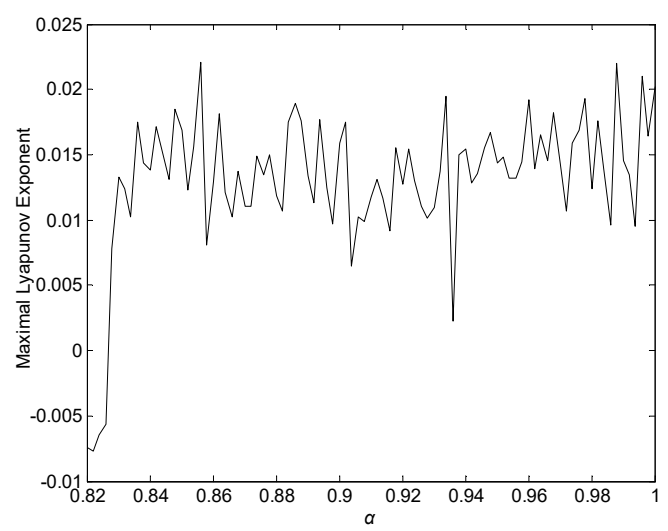

(a)

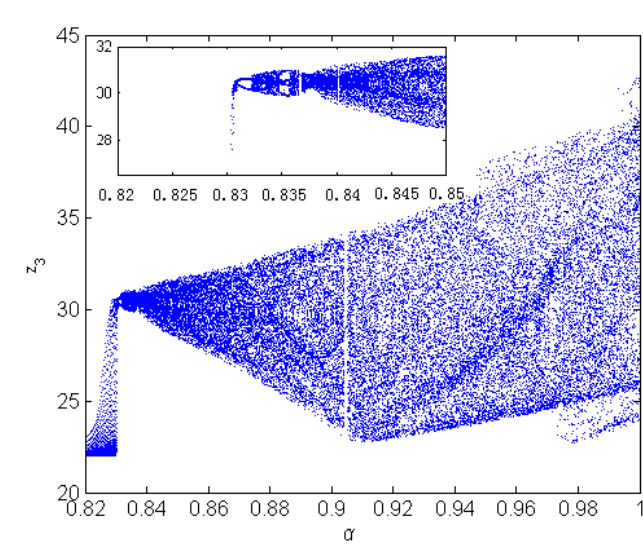

(b)

Figure 1. Dynamic behavior of fractional-order complex Chen system with commensurate order $\alpha(a=$ $30+i, b=3, c=26+0.6 i)$. (a) Maximal Lyapunov exponent spectrum. (b) The bifurcation diagram. 


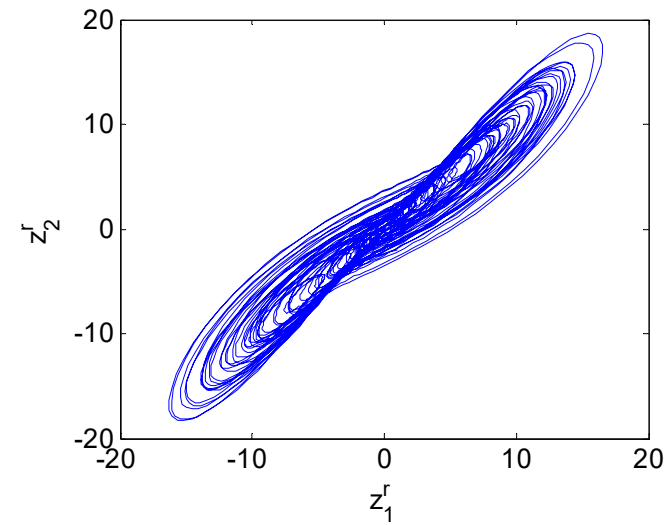

(a)

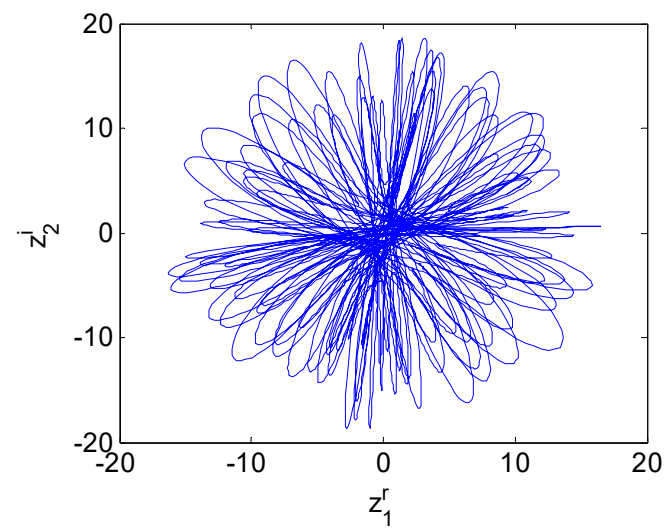

(c)

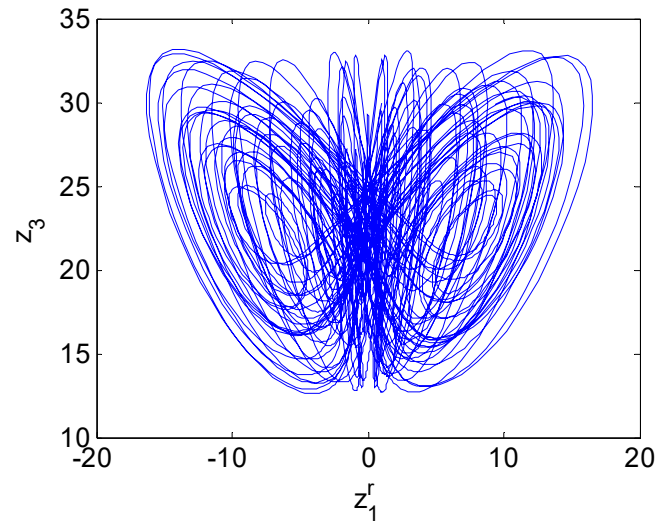

(b)

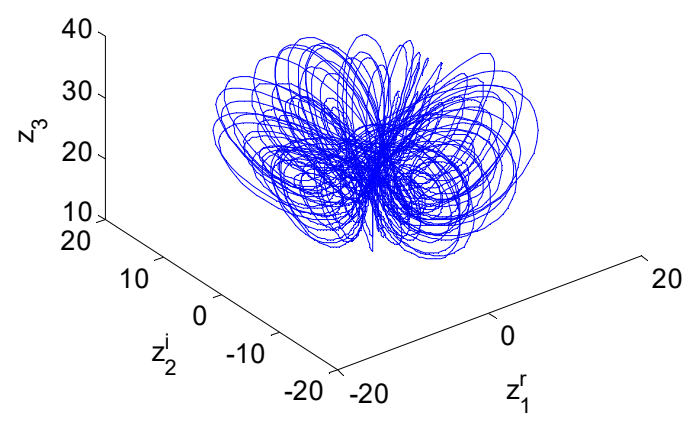

(d)

Figure 2. Chaotic attractors of fractional-order complex Chen system with $a=30+i, b=3, c=$ $26+0.6 i, \alpha=0.9$. (a) $z_{1}^{r}-z_{2^{\prime}}^{r}$ (b) $z_{1}^{r}-z_{3},(\mathbf{c}) z_{1}^{r}-z_{2^{\prime}}^{i}$ (d) $z_{1}^{r}-z_{2}^{i}-z_{3}$.

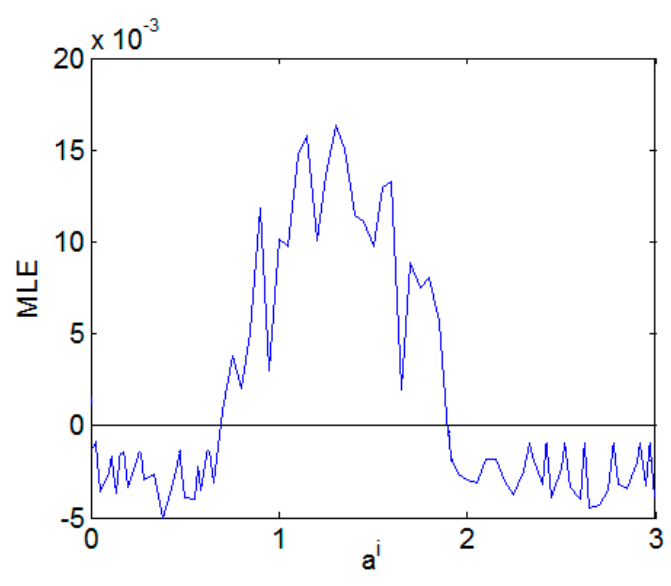

(a)

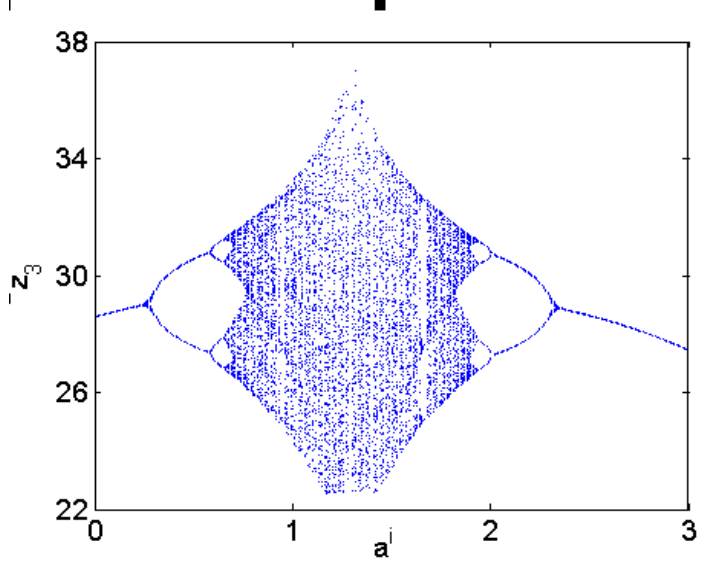

(b)

Figure 3. Dynamic behaviors of the fractional-order complex Chen System with varying $a^{i}\left(a^{r}=\right.$ $30+i, b=3, c=26+0.6 i, \alpha=0.95$ ). (a) Maximal Lyapunov Exponent. (b) Bifurcation diagram. 
Assuming the parameters $a, b, c$ are unknown, we select the complex scaling matrix $M=$ $\left(\begin{array}{ccc}1+i & 0 & 0 \\ 0 & 1-i & 0 \\ 0 & 0 & 1\end{array}\right)$. According to Theorem 1, we construct the response system as follows:

$$
\left(\begin{array}{l}
{ }_{0}^{C} D_{t}^{\alpha} w_{1} \\
{ }^{C} D_{t}^{\alpha} w_{2} \\
{ }^{C} D_{t}^{\alpha} w_{3}
\end{array}\right)=\left(\begin{array}{ccc}
w_{2} i-w_{1} & 0 & 0 \\
w_{1} i & w_{2}-w_{1} i & 0 \\
0 & 0 & -w_{3}
\end{array}\right)\left(\begin{array}{l}
\hat{a} \\
\hat{c} \\
\hat{b}
\end{array}\right)+\left(\begin{array}{c}
0 \\
w_{1} w_{3} i \\
\left(\bar{w}_{1} w_{2}-w_{1} \bar{w}_{2}\right) i / 2
\end{array}\right)+\left(\begin{array}{l}
u_{1} \\
u_{2} \\
u_{3}
\end{array}\right)
$$

where $\hat{a}, \hat{b}, \hat{c}$ are parameter estimations. $u_{1}, u_{2}, u_{3}$ are the controllers.

The error vector $e=\left(\begin{array}{lll}e_{1} & e_{2} & e_{3}\end{array}\right)^{T}=z-M w=\left(\begin{array}{c}z_{1}-(1+i) w_{1} \\ z_{2}-(1-i) w_{2} \\ z_{3}-w_{3}\end{array}\right)$ According to Theorem 1, the controllers and the update rules are selected as:

$$
\begin{gathered}
u_{1}=-k e_{1}, u_{2}=-k e_{2}, u_{3}=-k e_{3} \\
{ }_{0}^{C} D_{t}^{\alpha} k=\sigma e^{H} e=\sigma\left(\bar{e}_{1} e_{1}+\bar{e}_{2} e_{2}+\bar{e}_{3} e_{3}\right),(\sigma>0) \\
\left(\begin{array}{l}
C_{0}^{C} D_{t}^{\alpha} \hat{a} \\
{ }^{C} D_{t}^{\alpha} \hat{c} \\
{ }_{0}^{C} D_{t}^{\alpha} \hat{b}
\end{array}\right)=-\left(\begin{array}{ccc}
w_{2} i-w_{1} & 0 & 0 \\
w_{1} i & w_{2}-w_{1} i & 0 \\
0 & 0 & -w_{3}
\end{array}\right)^{H}\left(\begin{array}{l}
e_{1} \\
e_{2} \\
e_{3}
\end{array}\right)=\left(\begin{array}{c}
\left(\bar{w}_{2} i+\bar{w}_{1}\right) e_{1}+\bar{w}_{1} i e_{2} \\
-\left(\bar{w}_{2}+\bar{w}_{1} i\right) e_{2} \\
\bar{w}_{3} e_{3}
\end{array}\right)
\end{gathered}
$$

In the simulation, let $\alpha=0.9,(\boldsymbol{a}, \boldsymbol{b}, c)=(30+i, 3,26+0.6 i)$, the initial conditions $z(0)=$ $(1+i,-2-i, 6)^{T}, w(0)=(-1+i,-3+i, 10)^{T},(\hat{a}(0), \hat{b}(0), \hat{c}(0))=(20,2,20), k(0)=0$ and $\sigma=6$. Two systems can achieve CMPS and the parameters were identified, as shown in Figures 4 and 5.

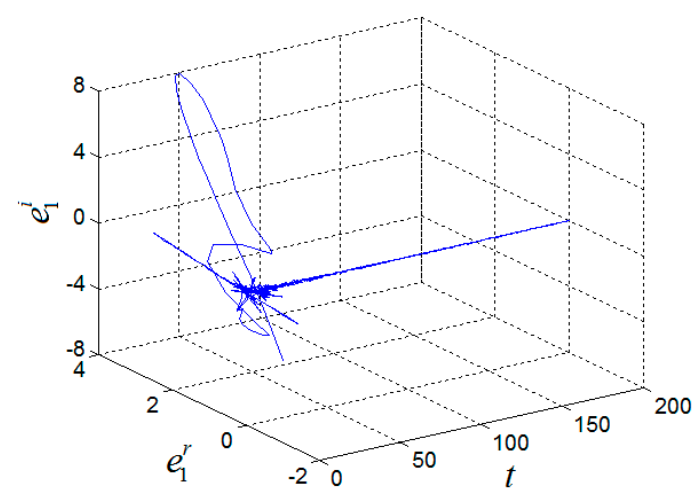

(a)

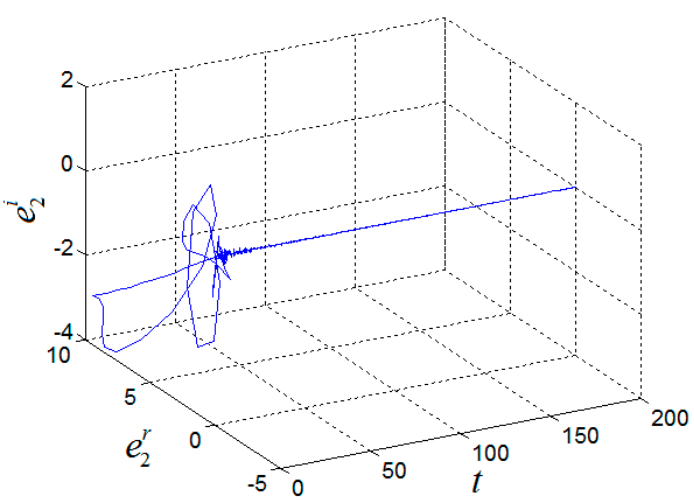

(b)

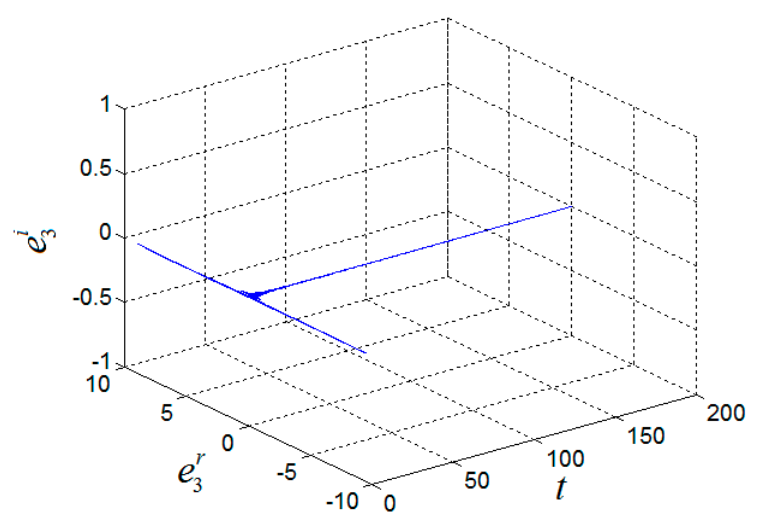

(c)

Figure 4. CMPS errors of the fractional-order complex Chen System. (a) $e_{1},(\mathbf{b}) e_{2},(\mathbf{c}) e_{3}$. 


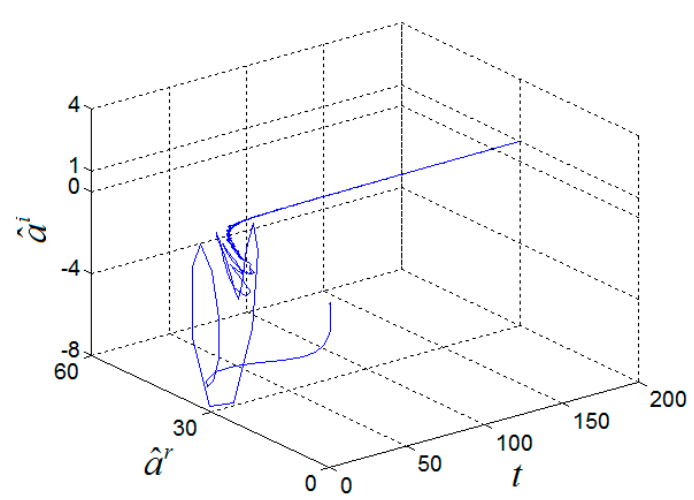

(a)

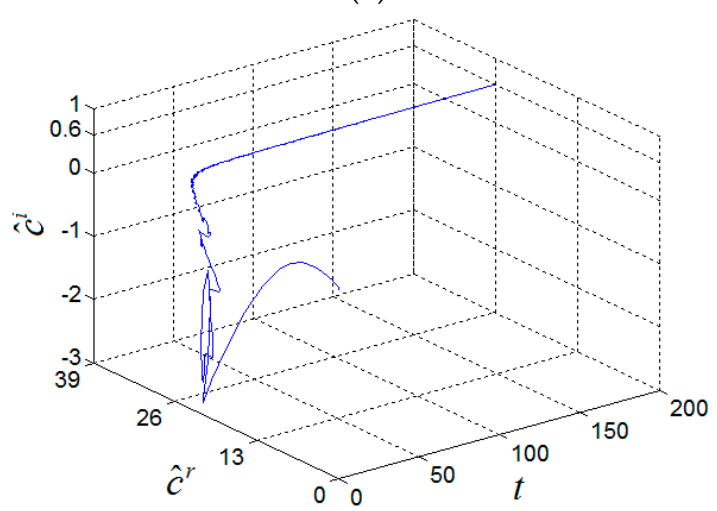

(c)

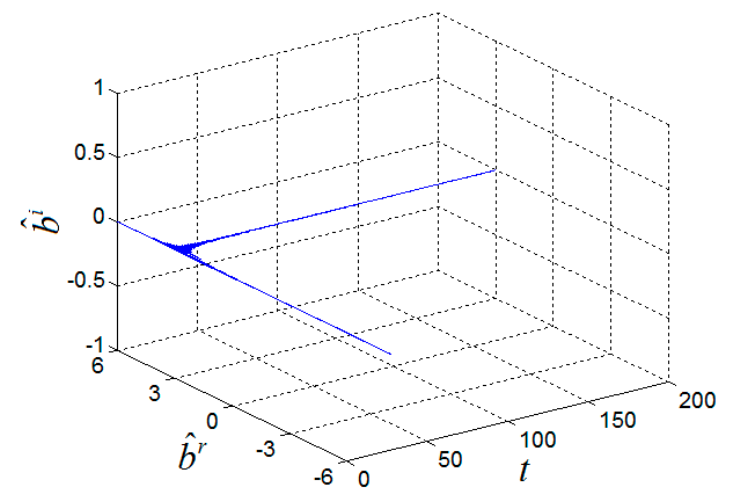

(b)

Figure 5. Estimated complex parameters of the fractional-order complex Chen System, (a) $\hat{a},(\mathbf{b}) \hat{b},(\mathbf{c}) \hat{c}$.

\section{Conclusions}

We study the CMPS of FOCCS with unknown complex parameters, and propose a method for analyzing FOCCS without separating systems into real and imaginary parts. By this method, the constructed response system can be asymptotically synchronized to an uncertain drive system with a desired complex scaling diagonal matrix. The proposed synchronization scheme retains the complex nature of fractional-order complex chaotic systems. It not only provides a new method of analyzing FOCCS but also significantly decreases the complexity of computation and analysis. In future works, we will further investigate the synchronization of FOCCS, and generalize the obtained results to more general cases, i.e., FOCCS with time delay and external disturbances.

Author Contributions: Conceptualization, R.Z.; software, S.F.; supervision, S.Y.

Funding: The author R.Z was supported by the Natural Science Foundation of Hebei province, China (No.A2015108010), and Key Research Projects of Xingtai university, China (No. XTXYZD2018002).

Conflicts of Interest: The authors declare no conflict of interest.

\section{References}

1. Gorenlo, R.; Mainardi, F. Fractional Calculus; Springer: Berlin, Germany, 1997.

2. Caponetto, R. Fractional Order Systems: Modeling and Control Applications; World Scientiic: Singapore, 2010; vol. 72.

3. Magin, R.L. Fractional Calculus in Bioengineering; Begell House Redding: New York, NY, USA, 2006.

4. Radwan, A.G.; Shamim, A.; Salama, K.N. Theory of fractional order elements based impedance matching networks. IEEE Microw Wirel Co. Lett. 2011, 21, 120-122. [CrossRef]

5. Atangana, A.; Gómez-Aguilar, J.F. A new derivative with normal distribution kernel: Theory, methods and applications. Physica A 2017, 476, 1-14. [CrossRef] 
6. Radwan, A.G.; Abd-El-Haiz, S.K.; Abdelhaleem, S.H. Image encryption in the fractional-order domain. In Proceedings of the 1st International Conference on Engineering and Technology, Cairo, Egypt, 10-11 October 2012.

7. Kiani-B, A.; Fallahi, K.; Pariz, N.; Leung, H. A chaotic secure communication scheme using fractional chaotic systems based on an extended fractional Kalman filter. Commun. Nonlinear Sci. Numer. Simul. 2009, 14, 863-879. [CrossRef]

8. Li, G.J.; Liu, H. Stability Analysis and Synchronization for a Class of Fractional-Order Neural Networks. Entropy 2016, 18, 55. [CrossRef]

9. Yin, C.; Cheng, Y.; Zhong, S.M.; Bai, Z. Fractional-order switching type control law design for adaptive sliding mode technique of 3D fractional-order nonlinear systems. Complexity 2016, 21, 363-373. [CrossRef]

10. Liu, H.; Li, S.G.; Wang, H.X.; Huo, Y.H.; Luo, J.H. Adaptive Synchronization for a Class of Uncertain Fractional-Order Neural Networks. Entropy 2015, 17, 7185-7200. [CrossRef]

11. Lu, J.G. Chaotic dynamics of the fractional-order Lü system and its synchronization. Phys. Lett. A 2006, 354, 305-311. [CrossRef]

12. Li, C.G.; Chen, G.R. Chaos in the fractional-order Chen system and it's control. Chaos Solitons Fractals 2004, 22, 549-554. [CrossRef]

13. Agrawal, S.K.; Srivastava, M.; Das, S. Synchronization of fractional order chaotic systems using active control method. Chaos Soliton Fractals 2012, 45, 737-752. [CrossRef]

14. Chen, L.P.; Chai, Y.; Wu, R.C. Lag projective synchronization in fractional-order chaotic (hyperchaotic) systems. Phys. Lett. A 2011, 375, 2099-2110. [CrossRef]

15. Zhang, Y.; Sun, J. Chaotic synchronization and anti-synchronization based on suitable separation. Phys. Lett. A 2004, 330, 442-447. [CrossRef]

16. Zhang, R.X.; Yang, S.P. Robust synchronization of two different fractional-order chaotic systems with unknown parameters using adaptive sliding mode approach. Nonlinear Dyn. 2013, 71, 269-278. [CrossRef]

17. Zhang, R.X.; Yang, S.P. Adaptive synchronization of fractional-order chaotic systems via a single driving variable. Nonlinear Dyn. 2011, 66, 831-837. [CrossRef]

18. Zhang, R.X.; Yang, S.P. Robust chaos synchronization of fractional-order chaotic systems with unknown parameters and uncertain perturbations. Nonlinear Dyn. 2012, 69, 983-992. [CrossRef]

19. Coronel-Escamilla, A.; Gómez-Aguilar, J.F.; Torres, L.; Escobar-Jiménez, R.F.; Valtierra-Rodríguez, M. Synchronization of chaotic systems involving fractional operators of Liouville-Caputo type with variable-order. Physica A 2017, 487, 1-21. [CrossRef]

20. Escalante-Martínez, J.E.; Gómez-Aguilar, J.F.; Calderón-Ramón, C.; Aguilar-Meléndez, A. Padilla-Longoria, P. A mathematical model of circadian rhythms synchronization using fractional differential equations system of coupled van der Pol oscillators. Int. J. Biomath. 2018, 11, 1-18. [CrossRef]

21. Hu, H.P.; Wang, J.K.; Xie, F.L. Dynamics Analysis of a New Fractional-Order Hopfield Neural Network with Delay and Its Generalized Projective Synchronization. Entropy 2019, 21, 1. [CrossRef]

22. Liu, L.; Du, C.; Zhang, X.; Li, J.; Shi, S. Adaptive Synchronization Strategy between Two Autonomous Dissipative Chaotic Systems Using Fractional-Order Mittag-Leffler Stability. Entropy 2019, 21, 383. [CrossRef]

23. Wang, S.; Wang, X.; Han, B. Complex Generalized Synchronization and Parameter Identification of Nonidentical Nonlinear Complex Systems. PLoS ONE 2016, 11, e0152099. [CrossRef]

24. Luo, C.; Wang, X. Chaos generated from the fractional-order complex Chen system and its application to digital secure communication. Int. J. Mod. Phys. C. 2013, 24, 72-77. [CrossRef]

25. Zhang, F.F.; Liu, S. Self-time-delay synchronization of time-delay coupled complex chaotic system and its applications to communication. In. J. Mod. Phys C 2014, 25, 559-583. [CrossRef]

26. Mahmoud, G.M.; Bountis, T. The dynamics of systems of complex nonlinear oscillators: A review. Int. J. Bifurc. Chaos 2004, 14, 3821-3846. [CrossRef]

27. Mahmoud, G.M.; Al-Kashif, M.A.; Farghaly, A.A. Chaotic and hyperchaotic attractors of a complex nonlinear system. J. Phys. A Math. Theor. 2008, 41, 055104. [CrossRef]

28. Roldab, E.; Devalcarcel, G.J.; Vilaseca, R. Single-mode-laser phase dynamics. Phys. Rev. A 1993, 48, 591-598. [CrossRef]

29. Ning, C.Z.; Haken, H. Detuned laser and the complex Lorenz equations-subcritical and supercritical Hopf bifurcations. Phys. Rev. A 1990, 41, 591-598. [CrossRef] 
30. Toronov, V.Y.; Derbov, V.L. Boundedness of attractors in the complex Lorenz model. Phys. Rev. E 1997, 55, 3689-3692. [CrossRef]

31. Luo, C.; Wang, X.Y. Chaos in the fractional-order complex Lorenz system and its synchronization. Nonlinear Dyn. 2013, 71, 241-257. [CrossRef]

32. Liu, X.J.; Hong, L.; Yang, L.X. Fractional-order complex T system: Bifurcations, chaos control, and synchronization. Nonlinear Dyn. 2014, 75, 589-602. [CrossRef]

33. Zhang, W.W.; Cao, J.D.; Chen, D.Y.; Alsaadi, F.E. Synchronization in fractional-order complex-valued delayed neural networks. Entropy 2018, 20, 54. [CrossRef]

34. Li, L.; Wang, Z.; Lu, J.W.; Li, Y.X. Adaptive Synchronization of Fractional-Order Complex-Valued Neural Networks with Discrete and Distributed Delays. Entropy 2018, 20, 124. [CrossRef]

35. Sun, J.; Deng, W.; Cui, G.Z.; Wang, Y.F. Real combination synchronization of three fractional-order complex-variable chaotic systems. Optik 2016, 127, 11460-11468. [CrossRef]

36. Yadav, V.K.; Srikanth, N.; Das, S. Dual function projective synchronization of fractional order complex chaotic systems. Optik 2016, 127, 10527-10538. [CrossRef]

37. Nian, F.Z.; Liu, X.M.; Zhang, Y.Q. Sliding mode synchronization of fractional-order complex chaotic system with parametric and external disturbances. Chaos Soliton Fractals 2018, 116, 22-28. [CrossRef]

38. Mahmoud, G.M.; Mahmoud, E.E. Complex modified projective synchronization of two chaotic complex nonlinear systems. Nonlinear Dyn. 2013, 73, 2231-2240. [CrossRef]

39. Xu, Q.; Xu, X.H.; Zhuang, S.X.; Xiao, J.X.; Song, C.H.; Che, C. New complex projective synchronization strategies for drive-response networks with fractional complex-variable dynamics. Appl. Math. Comput. 2018, 338, 552-566. [CrossRef]

40. Podlubny, I. Fractional Differential Equations; Academic Press: New York, NY, USA, 1999.

41. Aguila-Camacho, N.; Duarte-Mermoud, M.A.; Gallegos, J.A. Lyapunov functions for fractional order systems. Commun. Nonlinear Sci. Numer. Simulat. 2014, 19, 2951-2957. [CrossRef]

42. Xu, Q.; Zhuang, S.; Liu, S.; Xiao, J. Decentralized adaptive coupling synchronization of fractional-order complex-variable dynamical networks. Neurocomputing 2016, 186, 119-126. [CrossRef]

43. Bao, H.; Park, J.H.; Cao, J. Adaptive synchronization of fractional-order memristor-based neural networks with time delay. Nonlinear Dyn. 2015, 82, 1343-1354. [CrossRef]

44. Zhang, R.X.; Liu, Y.; Yang, S. Adaptive Synchronization of Fractional-Order Complex Chaotic system with Unknown Complex Parameters. Entropy 2019, 21, 207. [CrossRef]

45. Tao, F.; Sun, J.T. Stability of complex-valued impulsive system with delay. Appl. Math. Comput. 2014, 240, 102-108.

46. Wu, Z.Y.; Chen, G.R.; Fu, X.C. Synchronization of a network coupled with complex-variable chaotic systems. Chaos 2012, 22, 023127. [CrossRef] [PubMed]

47. Pano-Azucena, A.D.; Ovilla-Martinez, B.; Tlelo-Cuautle, E.; de la Fraga, L.G. FPGA-based implementation of different families of fractional-order chaotic oscillators applying Grounwald-Letnikov method. Commun. Nonlinear Sci. Numer. Simulat. 2019. [CrossRef]

48. Wolf, A.; Swift, J.B.; Swinney, H.L.; Vastano, J.A. Determining Lyapunov exponents from a time series. Physica D 1985, 16, 285-317. [CrossRef]

49. Carbajal-Gomez, V.H.; Tlelo-Cuautle, E.; Muñoz-Pacheco, J.M.; de la Fraga, L.G.; Sanchez-Lopez, C.; Fernandez-Fernandez, F.V. Optimization and CMOS design of chaotic oscillators robust to PVT variations: INVITED. Integr. VLSI J. 2018. [CrossRef]

(C) 2019 by the authors. Licensee MDPI, Basel, Switzerland. This article is an open access article distributed under the terms and conditions of the Creative Commons Attribution (CC BY) license (http://creativecommons.org/licenses/by/4.0/). 